\title{
A Portable Robot for Tele-rehabilitation: Remote Therapy and Outcome Evaluation
}

\author{
Park, Hyung-Soon, and Zhang, Li-Qun \\ Rehabilitation Institute of Chicago and Northwestern University \\ United States
}

\section{Introduction}

Neurological disorders including stoke, spinal cord injury, multiple sclerosis, and cerebral palsy affect the life of tens of millions of people worldwide. For those patients with motor impairment, physical therapy is the cornerstone in the rehabilitation process. A physical therapist uses training, exercises, and physical manipulation of the patient's body; however, physical therapy is labor intensive and generally requires manual and strenuous manipulation of the patient's limbs. Furthermore, different therapists may stretch the joint to different degrees, depending on his/her subjective judgment on the end-feeling, and they may also have different evaluation of the impairment, based on their experience and judgment. For these reasons, there is a need for a robotic device that can stretch the joint to its extreme positions with accurate and quantitative control of the resistance torque and stretching velocity.

The effective treatments and the monitoring of the progression of motor dysfunction typically relies upon a physical exam by an experienced clinician; however, for many

$\varepsilon$ individuals with motor impairment, routine access to expert clinical assessment is severely

0 limited by financial resources and distance to a qualified medical center, resulting in

$\stackrel{\Phi}{\equiv}$ suboptimal treatment therapies or dosages. The tele-rehabilitation robot system has been

ㄷ considered as a solution to this problem enabling remote delivery of rehabilitation and

O home care services for individuals with limited access to comprehensive medical and

$\stackrel{\Phi}{.}$ rehabilitation outpatient services.

Robotic devices have been utilized effectively in rehabilitation setups. Combining serial

casting with manual stretching is usually a more effective treatment for correcting ankle

\& plantar- or dorsi- flexion contracture (Moseley, 1997). Dynamic splinting and traction apply

I a continuous stretch to the joint involved through an adjustable spring mechanism

$\frac{\pi}{\pi}$ (Hepburn, 1987). The continuous passive motion (CPM) device is widely used in clinics and

in consumers' homes to move the joint within a pre-specified movement range, to prevent

\& postoperative adhesion and to reduce joint stiffness (Salter, 1989). The MIT-MANUS, a 2

¿ degree of freedom (DOF) robot, was developed to evaluate arm impairment quantitatively,

$\ll$ and to assist and guide patient's hand to reach a target in the arm workspace to enhance

Фे neurorehabilitation following brain injury (Krebs et al., 1998). The JavaTherapy system was

O used to guide patients to improve their motor skills by using a commercial force-feedback

Source: Rehabilitation Robotics, Book edited by Sashi S Kommu,

ISBN 978-3-902613-04-2, pp.648, Auqust 2007, Itech Education and Publishina, Vienna, Austria 
joystick connected to an orthopedic splint attached to the patient's wrist (Reinkensmeyer et al., 2002). The Rutgers Master II, an exoskeleton robot worn inside of the patient's hand, was used to increase hand strength in stroke patients (Popescu et al., 2000). The Virtual Driving Environment used a commercial force-feedback for gaming applications (Jadvah \& Krovi, 2004).

The tele-rehabilitation system developed in this study was aimed at both 'remote treatment' and 'remote outcome evaluation' by using a portable robot at home-based settings. The key factors in the realization of an effective rehabilitation device include the development of the required control technology to enable intelligent control for safe and effective treatment at a local clinic or patients' homes, the low-cost implementation to improve accessibility for those with limited resources, and improved portability of this device for enhanced mobility and user-friendliness.

\section{Task Definition}

Five tasks were defined for the therapeutic treatment as well as the outcome evaluation.

1) Passive Steretching Task: For therapeutic treatment, the spastic elbows of patients were stretched strenously to flexion and extension directions to loosen stiffened muscles. Patients sat upright with the shoulder abducted $20^{\circ}$. After adjusting the alignment between the elbow joint and the motor axis, the two extreme position limits in flexion and extension, and the torque limits were set. Then the device rotated the elbow about its flexion axis throughout its range of motion. The patients were asked to relax and not to react to the stretch. Each trial was two minutes long and about 20 trials were performed for one patient.

2) Passive range of motion Test: Before and after the strenous stretching, the passive range of motion was measured by the rotation angles within the torque limits. This task was similiar to the passive stretching task except for the values used for torque limits. Relatively smaller torque limits $(3 \mathrm{Nm})$ were used in passive range of motion test.

3) Active range of motion Test: The patients were asked to move the elbow voluntarily throughout the range of motion while the robotic device was controlled to be backdrivable. The elbow joint position during the movement was measured at the device.

4) Muscle Strength Test: The device was locked at a certain angle while the patients were asked to flex and extend their elbow with their maximum strength. The torque was measured at the device.

5) Spasticity Test: Spasticity was characterized by a velocity-dependent increase in tonic stretch reflexes which was considered to be associated with exaggerated stretch reflexes with a velocity-dependent increase in the resistance to passive movement (Lance, 1980). Furthermore, a 'catch' which is defined as sudden appearance of increased muscle tone could often be felt during passive movement of the spastic limb, and the catch angle was a commonly used clinical measure such as the Tardieu and Modified Tardieu Scales (Boyd \& Graham, 1999; Haugh et al., 2006). Therefore, the spasticity test was needed to characterize the velocity-dependent increase in 
resistance torque and the presence of 'catch'. Spasticity was evaluated by moving the patient's elbow with different velocities and measuring the differences in the resistance torques. The catch angle during the passive movement was determined as a clinical measure using a custom-developed method.

\section{System Design}

\subsection{Hardware Design}

An intelligent robotic device was developed to stretch the elbow and evaluate outcome (Fig. 1). A pre-made short-arm cast with padding was put on the wrist/forearm of the patients. Several sizes of the cast were made for different forearm sizes. The short-arm cast was then clamped to an aluminum beam that is mounted onto a servomotor through a torque sensor. The cast and forearm could be adjusted in the proximal-distal and medial-lateral directions to align the elbow flexion axis with the motor shaft. The motor was controlled by a data acquisition card $(6036 \mathrm{E}$, National Instruments Co.) installed in a laptop (Fig. 2). The position and the torque signals were collected by the card and a control program calculated corresponding commands. The commands were sent to the motor through the data acquisition card. The control system collected data at every one mili-second ( $1 \mathrm{kHz}$ of sampling rate).

For portability, the device was designed so that the weight of the device was less than $3 \mathrm{~kg}$ and the size was smaller than $14 \times 11.5 \times 20 \mathrm{~cm}^{3}$. The selection of a motor with higher weight-totorque ratio reduced the size and weight of the device. A small motor with speed reduction using a harmonic drive could achieve higher weight-to-torque ratio. The maximum torque of the device was chosen not to exceed $20 \mathrm{Nm}$ so that the device could be intrinsically safe but still had enough power to stretch the spastic elbow joint. Furthermore, a controller was embedded in the motor for compact design. The use of suction cups improved portability and easy installation of the device on any flat surfaces so that a patient could install the device independently at home environment. Upper arm and forearm supports were designed so that they could be adjusted conveniently. A three DOF ball joint was freely moved to adjust patient's upper arm and fixed at proper position by locking the ball joint. The upper arm support was designed to be adjustable in anterior-posterior and medial-lateral directions.

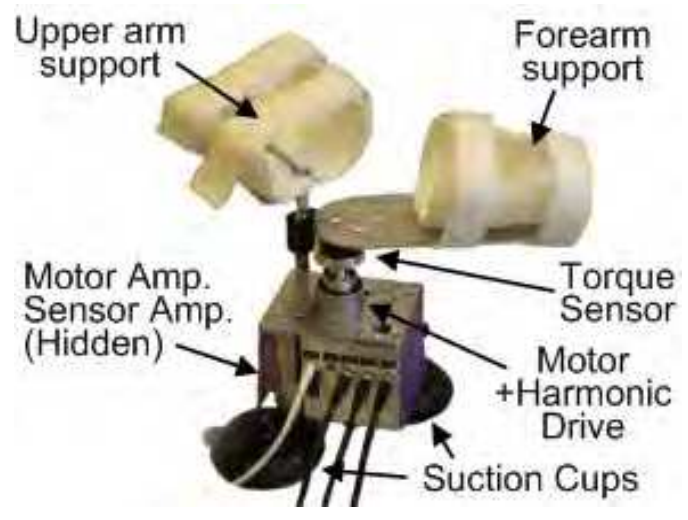

Fig. 1. A prototyped portable stretching device: The device designed to stretch elbow joint and evaluate treatment outcome in multiple aspects. The device is portable by using a small motor with built-in controller and suction cups. 


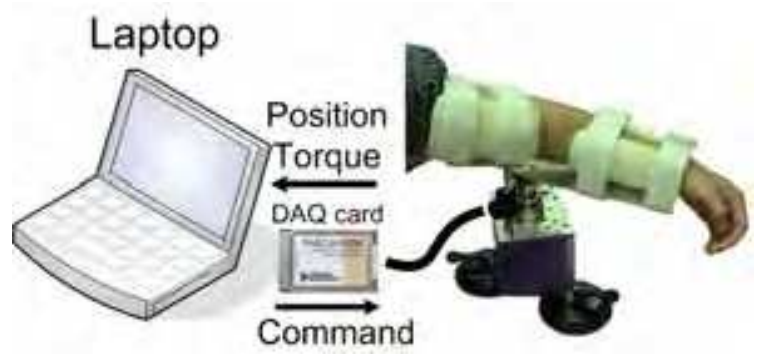

Fig. 2. Control diagram of the limb-stretching device: The device was controlled by a laptop for portability.

\subsection{Control System Design}

Two types of controllers were designed to perform the five tasks mentioned in Section 2. For the active range of motion test, the controller had to make the motor run back-drivable while, for other four tasks, the controller adjusted stretching speed based on the joint angle and joint torque.

The back-drivability was achieved by zero-torque regulation control (Fig. 3). The torque at the elbow joint was measured and fed back to create control commands to maintain zero torque at the elbow joint so that patients felt free when they voluntarily moved their elbow joints.

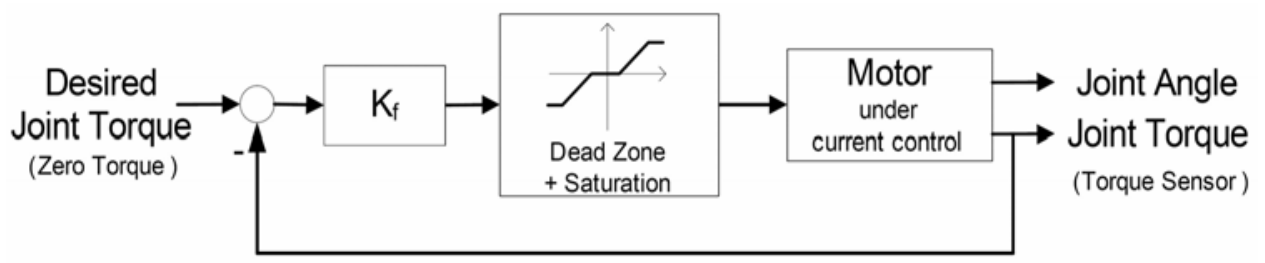

Fig. 3. Back-drivability controller for active range of motion test

The control structure for the passive stretching task, passive range of motion test, and spasticity test is shown in Fig. 4. For the passive stretching task, the stretching logic determined the direction of stretching. When the position or torque limits were reached, the logic switched the direction of stretching. With the direction of stretching determined, the intelligent velocity adjustment module adjusted the stretching velocity command, $V(t)$.

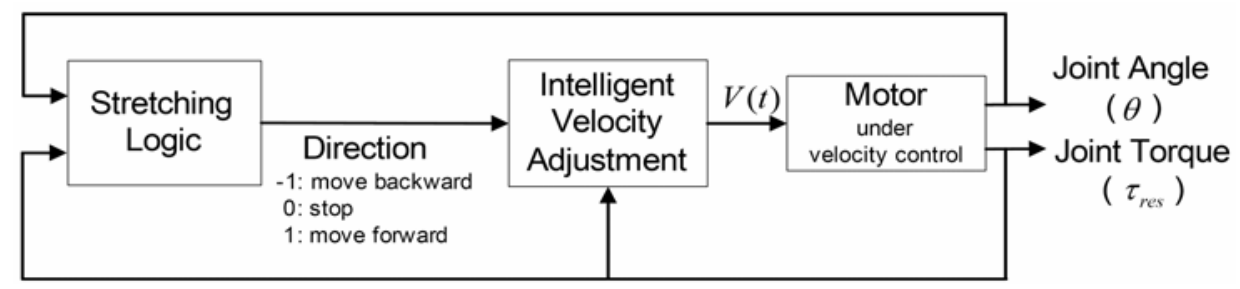

Fig. 4. Control structure at the passive stretching task

The flowchart in Fig. 5 explains the stretching logic in detail. The servomotor stretched the elbow within the range of motion until the time limit was reached. The range of motion was 
specified with the positive position limit $\left(\theta_{p}\right)$, the negative position limit $\left(\theta_{n}\right)$, and the additional further rotation angle $\left(\theta_{d}\right)$. When the position limits or the torque limits $\left(M_{p}\right.$ as the positive torque limit and $M_{n}$ as the negative torque limit) were reached, the servomotor held the joint at the limit position for $n$ seconds and rotated the elbow to the other direction.

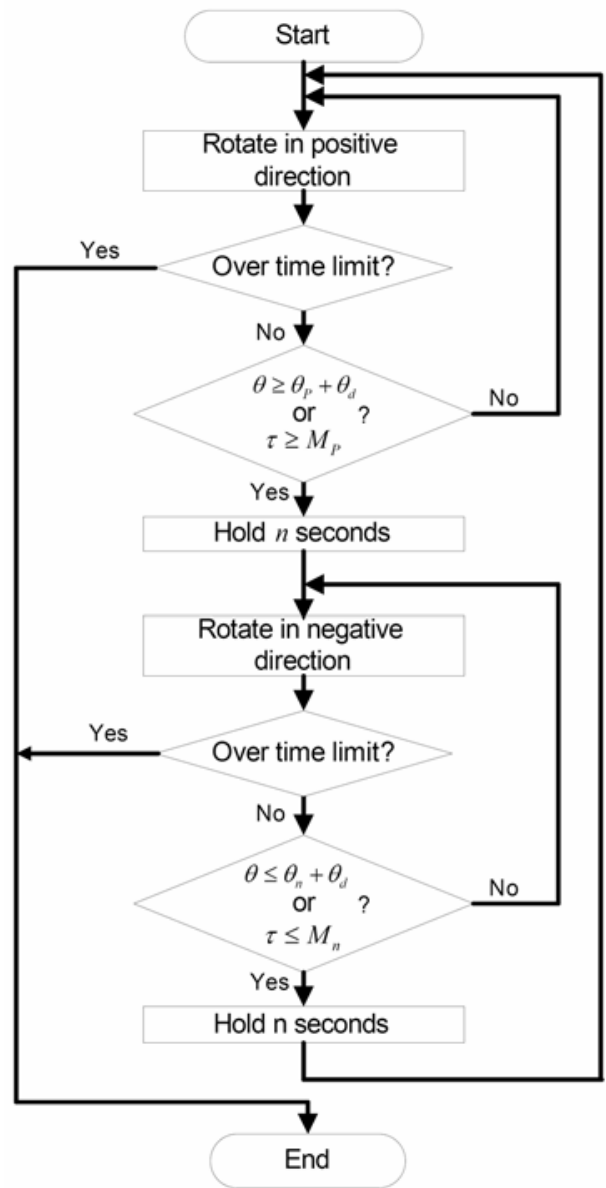

Fig. 5. Stretching logic for the passive stretching algorithm.

The intelligent stretching algorithm was developed so that, in the middle range of motion where resistance is low, the motor stretched the relatively slack muscles quickly and, near the extreme joint angles with increased resistance, the motor slowed down its speed in order to stretch the muscle-tendons slowly, resulting in a larger elbow range of motion (Zhang et. al., 2002). Once the specified peak resistance torque was reached, the motor held the joint at the extreme position for a period of time (e.g., $5 \mathrm{sec}$ ) and changed the direction of the motion. For safety, position limits were set by manually moving the joint to the extreme positions. The elbow flexion angle was monitored by the controller and room was left beyond the position limits $\left(\theta_{d}\right)$ to allow stretching-induced improvement. The controller adjusted the motor velocity $(V(t))$ at every one mili-second according to the following rules: 


$$
V(t)=\left\{\begin{array}{cc}
0, & \operatorname{if}\left(\tau_{\text {res }} \geq M_{p} \text { or } \theta \geq \theta_{p}+\theta_{d}\right) \text { and need to hold } \\
-V_{\max }, & \operatorname{if}\left(\tau_{\text {res }} \geq M_{p} \text { or } \theta \geq \theta_{p}+\theta_{d}\right) \text { and have held long enough } \\
\max \left(\frac{C}{\tau_{\text {res }}(t)}, V_{\min }\right), & \text { if } 0<\tau_{\text {res }}<M_{p} \\
\min \left(\frac{C}{\tau_{\text {res }}(t)},-V_{\min }\right), & \text { if }-M_{p}<\tau_{\text {res }}<0 \\
V_{\max }, & \operatorname{if}\left(\tau_{\text {res }} \leq-M_{n} \text { or } \theta \leq \theta_{n}-\theta_{d}\right) \text { and have held long enough } \\
0, & \operatorname{if}\left(\tau_{\text {res }} \leq-M_{n} \text { or } \theta \leq \theta_{n}-\theta_{d}\right) \text { and need to hold }
\end{array}\right.
$$

where $\theta(t)$ and $\tau_{\text {res }}(t)$ represent the elbow position and resistance torque at time $t$, respectively. $V_{\min }$ and $V_{\max }$ represent the magnitudes of the minimum and maximum speed, respectively. $C$ is a constant, scaling the $1 / \tau_{\text {res }}(t)$ to the appropriate stretching velocity. When $\theta_{d}$ was chosen to be a very large number to allow the device move beyond the position limits or when $\theta_{p}$ and $\theta_{n}$ were set outside the range of motion, the stretching control was dominated by the resistance torque (certainly the stretching was safe) and the motor reversed its rotation once the specific resistance torque was reached for the specific amount of time. On the other hand, when $M_{p}$ and $M_{n}$ were chosen to be very large, the stretching was restricted by the position limits. In general, we wanted the stretching reached the torque limits at both ends of the range of motion with the position limits incorporated into the control scheme as a safety measure and as an optional mode of stretching, therefore the $\theta_{p}$ and $\theta_{n}$ were set to approximately match the range of motion by manually pushing the joint to its extreme positions and the $\theta_{d}$ was chosen as a positive number (e.g., $5^{\circ}$ ). In this way, the torque limits were reached most of the time, while the position limits still restricted potential excessive elbow movement. All the control parameters could be changed conveniently within pre-specified ranges.

For safety, the controller checked the joint position and torque signals at every one milisecond and shutdown the system if they were out of pre-specified ranges. Mechanical and electrical stops were used to restrict the motor range of motion. The subject had a stop switch so that he/she could shutdown the motor by pressing the switch.

The same controller could be used to perform the other tasks such as passive range of motion test, muscle strength test, and spasticity test. For the passive range of motion test, same controller with lower torque limits $(3 \mathrm{Nm})$ was used. For muscle strength test, zero velocity commands were sent out to hold the joint at a certain rotation angle. For spasticity tests, same controller but with different values of $V_{\max }$ (ranging from $30^{\circ} / \mathrm{sec}$ to $210^{\circ} / \mathrm{sec}$ ) was used to observe the velocity dependence of resistance torque.

\section{Experimental Results}

Patients post stroke joined the study and they were tested at Rehabilitation Institute of Chicago with the experimental procedures approved by Institutional Review Board. All subjects gave the consent forms and performed the five tasks described in Section 2.

1) Passive Stretching Task: Subjects sat upright with the shoulder abducted $20^{\circ}$. After adjusting the alignment between the elbow joint and the motor axis, the two extreme position limits $\left(\theta_{p}\right.$ and $\left.\theta_{n}\right)$, the torque limits $\left(M_{p}\right.$ and $\left.M_{n}\right)$ and $\theta_{d}$ were set. Then the device rotated the elbow about its flexion axis throughout its range of motion by following the algorithm described in Fig. 5. The subjects were asked to relax and not to react to the stretch. The maximum stretching 
velocity was up to $20^{\circ} / \mathrm{sec}$ and it was only possible at middle range of motion due to the control strategy. Peak resistance torque (typical values: 5 9 Nm), and length of the holding period (typical value: $3 \mathrm{sec}$ ) at the joint extreme positions was specified and if needed, they were adjusted conveniently for each trial. Each trial was two minutes long and about 20 trials were performed for one subject. Elbow flexion angle $\theta(t)$ and joint torque $\tau_{\text {res }}(t)$ was recorded. All the signals were low-pass filtered and sampled at $1 \mathrm{kHz}$.

The spastic elbow joints of stroke patients were stretched to extreme flexion and extension repeatedly with the stretching velocity controlled based on the resistance torque. The velocity was reduced near the extreme positions and was inversely proportional to the resistance torque (Fig. 6).
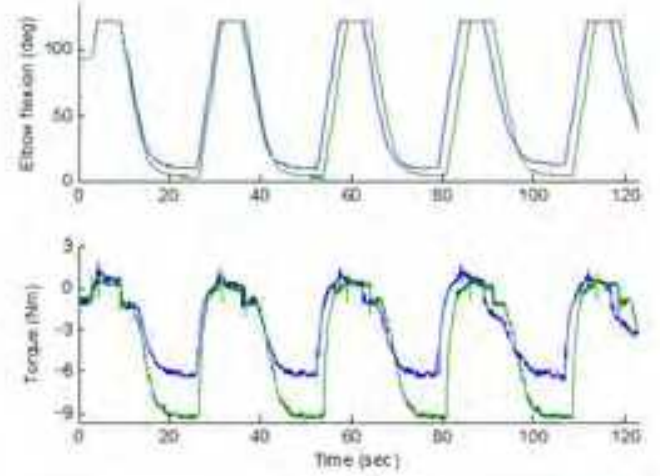

Fig. 6. Elbow flexion angle and torque during two stretching trials ( $6 \& 9 \mathrm{Nm}$ peak torque) in a spastic elbow. The stretching velocity was reduced gradually down to 0 as the resistance increased. Position limit was reached at the extreme flexion, because the joint was not stiff in flexion direction.

After the 40 minutes passive stretching session, the patients felt good about the forceful stretching. The strenuous stretching have loosened up their stiff elbow and increased the range of motion. In a subject post-stroke, the controlled forceful stretching increased the passive range of motion from $18-129^{\circ}$ flexion to $3-136^{\circ}$ flexion. Since the strenuous stretching loosened the stiff elbow joint, the patient was able to extend the elbow voluntarily to $10^{\circ}$ flexion compared with $20^{\circ}$ before stretching. Functionally, patients could raise the hand to reach larger range of motion. For the same patient with severe spasticity, the patient could move the hand upward in front of the body by $18 \mathrm{~cm}$, which was increased to $28 \mathrm{~cm}$ after the intelligent stretching with reduced biceps/triceps co-contraction. It seems the stretching had some lasting effect, probably related to the lack of strenuous stretching due to the difficulties involved. A couple of days after stretching a stroke patient happily raised both arms to show us the improvement he could tell obviously.

2) Passive range of motion test: Passive range of motion was measured using the same control method in the passive stretching task except for the smaller torque limits $\left(M_{p}=3 \mathrm{Nm}, M_{n}=-\right.$ $3 \mathrm{Nm}$ ). The flexion angles at the peak torques were measured before and after the strenuous stretching to evaluate improvement in the passive range of motion.

3) Active range of motion test: The subjects were asked to voluantarily move their elbow joint in both extension and flexion direction with the device controlled back-drivable. The maximum and minimum flexion angles were measured during the voluntary movement (Fig. 7). 

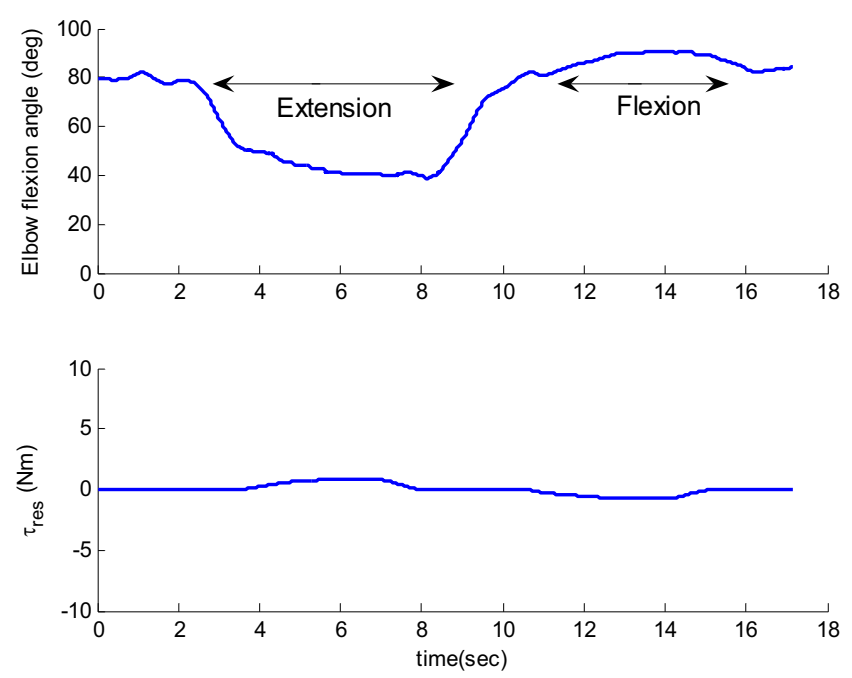

Fig. 7. Active range of motion test (typical data).

4) Muscle strength test: The subjects were asked to flex and extend their elbows with their maximum strength with the robotic device locked at $90^{\circ}$ flexion angle. The peak torques were measured for the evaluation of the muscle strength (Fig. 8).
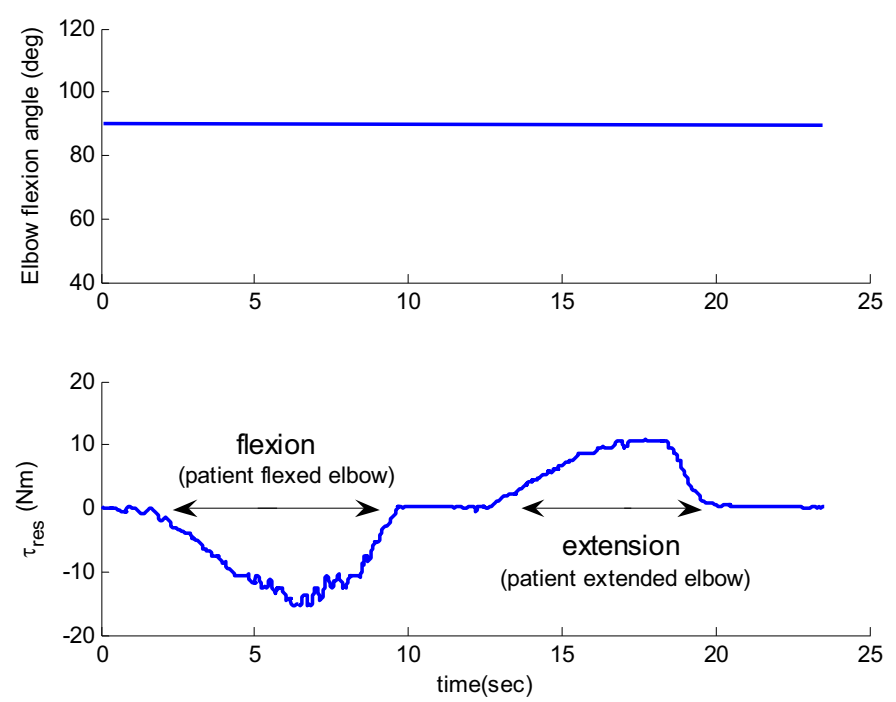

Fig. 8. Muscle strength test (typical data)

5) Spasticity test: The spastic elbows were stretched with slow $\left(30^{\circ} / \mathrm{sec}\right)$, medium $\left(90^{\circ} / \mathrm{sec}\right)$, and fast $\left(180^{\circ} / \mathrm{sec}\right)$ velocities to extension direction and the resultant flexion angles and the resistance torques were measured during the stretch. 
Velocity dependence of the resistance torque was observed from the collected data. Higher joint stiffness was measured for higher extension speeds (Fig. 9).

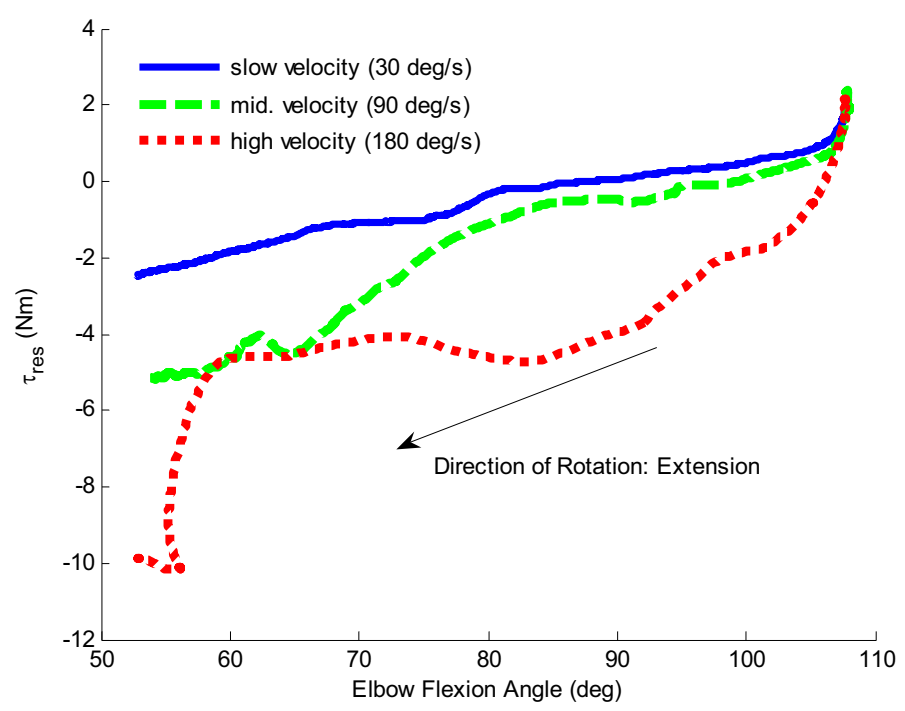

Fig. 9. Velocity dependence of resistance torque. Higher stiffness was measured for higher speeds.

There were two events associated with the 'catch angle' commonly measured in clinical practice.

1 The angle where the derivative of the resistance torque $\left(d \tau_{\text {res }} / d t\right)$ reached the peak which was related to the rapid increase in the resistance torque due to the stretch reflex action. The peak of $d \tau_{\text {res }} / d t$ was used to determine the catch angle (Peng et al., 2004; Wu et al., 2006).

2 The angle where the clinician slowed the movement speed to a local minimum after he/she felt the rapid increase in resistance. This angle with the local minimum speed was reached shortly after the instant corresponding to the peak $d \tau_{\text {res }} / d t$ and catch angle. It was used as a landmark to help determine the location of the peak of $d \tau_{\text {res }} / d t$.

In the spasticity test, there were two issues important for the safety of the patients. First, the robot might move the elbow beyond the range of motioin. Second, the robot could not slow the speed of stretching when strong reflex action occurred whereas, during an assessment with clinician, he/she might slow the speed of stretching in reaction to a 'catch' associated with a rapid increase of the resistance. For the first issue, the robotic device was programmed not to stretch beyond the patient's range of motion which was measured during previous passive range of motiono test. For the second issue, the robot simulated the clinician's reaction to the feel of catch. The robot slowed the stretching speed inversely proportional to the resistance torque after the rapid increase in $d \tau_{\text {res }} / d t$ was detected (Fig. 10). 

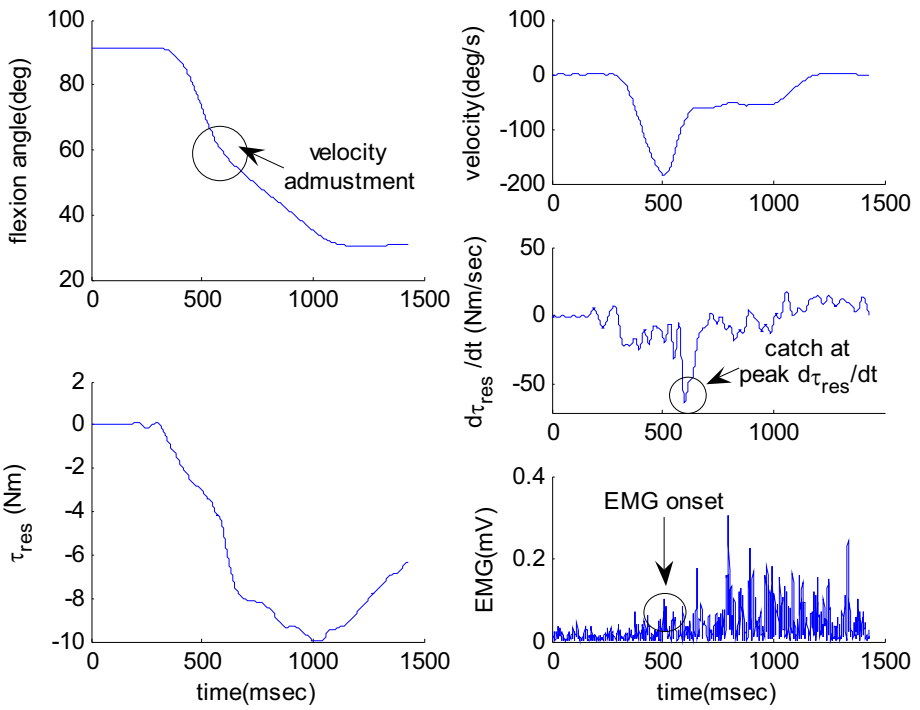

Fig. 10. The catch angle measurement. The robot stretched the patient's elbow within the range of motion. The stretching speed slowed down after the robot detected the peak $d \tau_{\text {res }} / d t$ at 580 msec. Electromyography (EMG) signal was monitored to confirm the stretch reflex action occurred around $500 \mathrm{msec}$. After the biceps EMG was onset, the peak value of $d \tau_{\text {res }} / d t$ was observed at $580 \mathrm{msec}$ with the flexion angle at $58.6^{\circ}$.

\section{Conclusions}

A portable rehabilitation robot for the elbow joint of neurologically impaired patients was developed to perform physical treatment task followed by the outcome evaluation for monitoring the progression of the therapy.

The hardware was designed for portability by using the small actuator and compact electronic components. After defining five tasks, two controllers were developed and implemented accordingly. For the active range of motion test, a torque controller maintaining zero torque was developed to make the device back-drivable. For other tests, velocity controller with intelligent velocity adjustment algorithm was developed in order to stretch the spastic/contractured elbow joint forcefully and safely.

The sequence of the tasks was organized to insure patient safety. The passive range of motion test was performed at the beginning of the tele-rehabilitation session to set position/torque limits of the patient's elbow. The limit values were then used in other tasks to insure that the joint was moved within the safe range.

The tele-rehabilitation robot developed in this study enabled the remote therapy followed by monitoring of progression of physical treatment. After remote treatment by rehabilitative robots, the progression of the physical treatment could be monitored remotely through audio-visual media using video-conferencing techniques (Brennan et al., 2004; Lai et al., 2004). In addition to the audio-visual data, this study could add quantitative physical measures such as the passive/active range of motion, muscle strength and spasticity related 
phenomenon - the velocity dependence of resistance torque and the catch angle. These quantitative measures made the remote physical examination more accurate and closer to an in-person physical examination.

Considering the nationwide shortage of therapists, the tele-rehabilitation system can complement the therapist's treatment and give patients more choices for the different treatment options. Potentially, it can be used by a therapist in a local clinic to treat more patients simultaneously with objective measurement and control.

Tele-rehabilitation has attracted much attention due to its economic benefits (Palsbo, 2004) saving both time and costs involved in rehabilitation. The simple yet intelligent design and relatively low-cost of the portable tele-rehabilitation system can potentially be used at home for convenient treatment with initial instruction/training by a therapist, which may make the potential market large and valuable.

Based on this study, similar portable stretching devices can be developed for other joints such as ankle, wrist, fingers, and knee joints. The single DOF device can also be extended to multi-DOF device to cover more complex tasks including shoulder movements, forearm twisting, wrist motions, and finger motions. Furthermore, the intelligent stretching can be similarly applied to other neurologically impaired populations such as spinal cord injury, multiple sclerosis, and cerebral palsy having similar problems with their spastic/contractured joints, and orthopaedic patients for whom continuous passive motion machines are widely used.

\section{Acknowledgement}

This material is based upon work supported by the U.S. National Science Foundation and National Institute of Health.

\section{References}

Boyd, R. \& Graham, H. (1999). Objective measurement of clinical findings in the use of botulinum toxin type A for the management of children with cerebral palsy. Eur J Neurol, 6, 4, 23-35.

Brennan, D.; Georgeadis, A.; Baron, C. \& Barker, L. (2004). The effect of videoconferencebased telerehabilitation on story retelling performance by brain-injured subjects and its implications for remote speech-language therapy. Telemedicine Journal and E-Health, 10, 2, 147-154.

Haugh, A.; Pandyan, A. \& Johnson, G. (2006). A systematic review of the Tardieu Scale for the measurement of spasticity. Disabil Rehabil, 28, 15, 899-907.

Hepburn, G. (1987). Case studies: contracture and stiff joint management with Dynasplint. J. Orthop. Sports Physical Therapy, 8, 498-504.

Jadvah, C. \& Krovi, V. (2004). A low-cost framework for individualized interactive telerehabilitation. Proceedings of IEEE Int. Conf. in EMBS, pp. 3297-3300, San Francisco CA, Sep. 2004, IEEE, New York.

Krebs, H.; Hogan, N.; Aisen, M. \& Volpe, B. (1998). Robot-Aided Neurorehabilitation. IEEE Trans. on Rehabilitation Engineering, 6, 75-87.

Lai, J.;Woo, J.; Hui, E. \& Chan, W. (2004). Telerehabilitation - a new model for community-based stroke rehabilitation. Journal of Telemedicine and Telecare, 10 , 4, 199-205. 
Lance, J. (1980). Spasticity: Disordered Motor Control, Year Book Medical Publishers, Chicago.

Moseley, A. (1997). The effect of casting combined with stretching on passive ankle dorsiflexion in adults with traumatic head injuries. Physical Therapy, 77, 240-247.

Palsbo, S. (2004). Medicaid payment for telerehabilitation. Archives of Physical Medicine and Rehabilitation, 85, 7, 1188-1191.

Peng, Q.; Shah, P.; Selles, R.; Gaebler-Spira, D. \& Zhang, L. (2004). Measurement of ankle spasticity in children with cerebral palsy using a manual spasticity evaluator. Proceedings of International Conference in IEEE EMBS, pp. 4896-4899, San Francisco CA, Sep. 2004, IEEE, New York.

Popescu, V.; Burdea, G.; Bouzit, M. \& Hentz, V. (2000). A virtual-reality-based telerehabilitation system with force feedback. IEEE Transactions on Information Technology in Biomedicine, 4, 1, 45-51.

Reinkensmeyer, D.; Pang, C.; Nessler, J. \& Painter, C. (2002). Web-based telerehabilitation for the upper extremity after stroke. IEEE Transactions on Neural Systems and Rehabilitation Engineering, 10, 2, 102-108.

Salter, R. (1989). The biological concept of continuous passive motion of synovial joints. Clinical Orthopedics and Related Research, 242, 12-25.

Wu, Y.; Park, H.; Ren, Y; Gaebler, D; Chen, J.; Roth, E. \& Zhang, L. (2006). Measurement of Elbow Spasticity in Stroke Patients Using a Manual Spasticity Evaluator. Proceedings of IEEE Int. Conf. in EMBS, pp. 3974-3977, New York NY, Sep. 2006, IEEE, New York.

Zhang, L. et. al. (2002). Intelligent stretching for ankle joints with contracture/spasticity. IEEE Trans. Neural System and Rehabilitation Engineering, 10, 3, 149-157. 


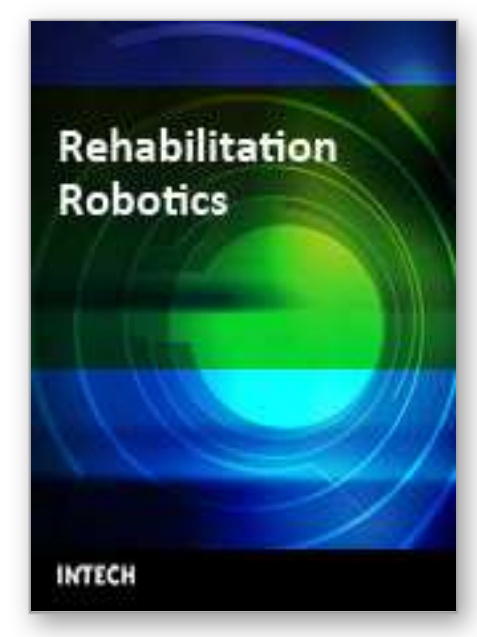

\author{
Rehabilitation Robotics \\ Edited by Sashi S Kommu
}

ISBN 978-3-902613-04-2

Hard cover, 648 pages

Publisher I-Tech Education and Publishing

Published online 01, August, 2007

Published in print edition August, 2007

The coupling of several areas of the medical field with recent advances in robotic systems has seen a paradigm shift in our approach to selected sectors of medical care, especially over the last decade.

Rehabilitation medicine is one such area. The development of advanced robotic systems has ushered with it an exponential number of trials and experiments aimed at optimising restoration of quality of life to those who are physically debilitated. Despite these developments, there remains a paucity in the presentation of these advances in the form of a comprehensive tool. This book was written to present the most recent advances in rehabilitation robotics known to date from the perspective of some of the leading experts in the field and presents an interesting array of developments put into 33 comprehensive chapters. The chapters are presented in a way that the reader will get a seamless impression of the current concepts of optimal modes of both experimental and ap- plicable roles of robotic devices.

\title{
How to reference
}

In order to correctly reference this scholarly work, feel free to copy and paste the following:

Park, Hyung-Soon, and Zhang, Li-Qun (2007). A Portable Robot for Tele-Rehabilitation: Remote Therapy and Outcome Evaluation, Rehabilitation Robotics, Sashi S Kommu (Ed.), ISBN: 978-3-902613-04-2, InTech, Available from: http://www.intechopen.com/books/rehabilitation_robotics/a_portable_robot_for_telerehabilitation_remote_therapy_and_outcome_evaluation

\section{INTECH}

open science | open minds

\author{
InTech Europe \\ University Campus STeP Ri \\ Slavka Krautzeka 83/A \\ 51000 Rijeka, Croatia \\ Phone: +385 (51) 770447 \\ Fax: +385 (51) 686166 \\ www.intechopen.com
}

\author{
InTech China \\ Unit 405, Office Block, Hotel Equatorial Shanghai \\ No.65, Yan An Road (West), Shanghai, 200040, China \\ 中国上海市延安西路65号上海国际贵都大饭店办公楼405单元 \\ Phone: +86-21-62489820 \\ Fax: $+86-21-62489821$
}


(C) 2007 The Author(s). Licensee IntechOpen. This chapter is distributed under the terms of the Creative Commons Attribution-NonCommercial-ShareAlike-3.0 License, which permits use, distribution and reproduction for non-commercial purposes, provided the original is properly cited and derivative works building on this content are distributed under the same license. 